\title{
Interpréter la sériation des problèmes des Arithmétiques de Diophante : une forme de modélisation ? ${ }^{1}$
}

\author{
Alain Bernard ${ }^{\mathrm{a}}$ \\ Centre Koyré, 27 rue Damesme, 75013 Paris, Labex HASTEC ANR-10-LABX-85, France
}

\begin{abstract}
Résumé. Dans cette étude on propose une réflexion méthodologique sur une première tentative de rendre compte de la progressivité des premiers problèmes arithmétiques contenus dans le recueil ancien des Arithmétiques du à Diophante d'Alexandrie. On résume le principe du cadre analytique que Jean Christianidis et moi-même avons bâti pour rendre compte de cette progressivité, en le rapportant au questionnement initial qui l'a motivé. On montre en outre que l'intérêt de cette interprétation réside au moins autant dans ses succès (exhiber une forme de progressivité qui n'est pas évidente a priori) que dans ses limites, dont l'identification conduit à explorer et définir de nouvelles pistes d'interprétation. L'ensemble de ce processus est finalement interprété comme un processus de modélisation, au sens où l'entend Willard McCarty dans son étude Humanities Computing (2005). Cette explicitation conduit également à distinguer différentes formes de sérialité, notamment déclarative (explicitée par Diophante), et reconstruite (via les cadres analytiques proposés) : l'effort de modélisation vise précisément à réduire l'écart entre ces deux types.
\end{abstract}

\begin{abstract}
Interpreting the ordering of problems in Diophantus's Arithmetica: A way to build a model of the text? The present study proposes a methodological reflection on a first attempt to account for the progression of the first problems contained in an ancient treatise, the Arithmetica of Diophantus of Alexandria. I first summarize the basic principles of the analytical framework that Jean Christianidis and I have built for this purpose and I relate them to the initial questions that motivated this attempt. I then show that the interest of such an interpretation lies not only in its alleged successes (to show indeed that there is in Diophatnus's problems a form of progressivity) but also in its limits. For, identifying the latter amounts to exploring and defining new interpretative possibilities. The whole process is finally interpreted in terms of a modeling process, following the seminal ideas of Willard McCarty in his 2005 book Humanities Computing. This explanation also leads me to distinguish between two particular types of seriality. On the one hand we have the declarative seriality, such as that expressed by Diophantus himself in his preface, and on the other reconstructed seriality, which is intrinsic to any attempt to build a framework of interpretation like the one we proposed. The attempt at building models can be seen as a way to reduce the gap between the two.
\end{abstract}

\footnotetext{
a e-mail : alain.bernard@u-pec.fr

${ }^{1}$ Je remercie Bernard Vitrac, Jean Christianidis et Aurélien Berra pour leurs remarques détaillées sur ce texte, qui m'ont permis de considérablement l'améliorer.
}

This is an Open Access article distributed under the terms of the Creative Commons Attribution License 4.0, which permits unrestricted use, distribution, and reproduction in any medium, provided the original work is properly cited. 


\section{SHS Web of Conferences}

\section{Introduction}

Cette contribution propose une réflexion méthodologique sur l'interprétation possible des problèmes qui forment les trois premiers livres des Arithmétiques de Diophante. Ce traité mathématique ancien de haut niveau, initialement constitué de treize livres de problèmes arithmétiques introduits par une longue préface, constitue un objet intéressant pour l'histoire culturelle des mathématiques anciennes. En effet, une étude séminale de Jean Christianidis (2007) introduisait la possibilité d'interpréter la cohérence du traité de Diophante dans les termes de la culture rhétorique ancienne, dont j'avais déjà indiqué la prégnance pour d'autres traités anciens, notamment la Collection mathématique de Pappus d'Alexandrie (Bernard, 2003), ou encore le commentaire de Proclus aux Éléments d'Euclide (Bernard, 2010). L'étude de Christianidis suggérait en effet pour la première fois qu'il soit possible d'interpréter en termes rhétoriques la notion d'invention qui sert d'exergue à la préface de Diophante (Tableau 1).

Tableau 1. Les premières lignes des Arithmétiques (Arithm. 13.3-7).

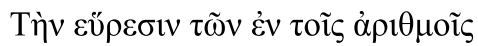
$\pi \rho \circ \beta \lambda \eta \mu \alpha ́ \tau \omega v, \tau 1 \mu 1 \omega ́ \tau \alpha \tau \dot{\varepsilon} \mu \mathrm{ol} \Delta \mathrm{tov} \sigma \dot{\sigma \varepsilon} \varepsilon$,

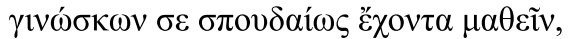

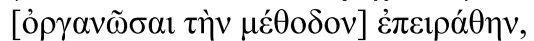

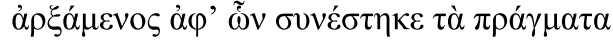

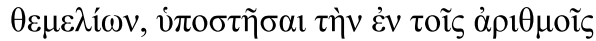

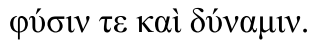

Quant à l'invention des < solutions de $>$ problèmes de nombres, mon très honoré Dionysius, sachant que tu es impatient de l'apprendre ${ }^{2}$, j'ai entrepris d'exposer la nature et la puissance dans les nombres, en commençant par les fondements, sur lesquels ces choses sont établies.

J. Christianidis propose dans le même article une première interprétation des solutions diophantiennes en termes d'invention et de disposition (2007, Sect. 3.3). La discussion autour de l'idée séminale d'une « interprétation rhétorique » du traité diophantien a également servi de point de départ d'un travail que. Christianidis et moi-même avons engagé à partir de 2006 et qui a aboutit début 2012 à une publication présentant un nouveau cadre analytique pour l'interprétation de la progressivité des trois premiers livres des problèmes diophantiens (Bernard \& Christianidis, 2012). On verra plus loin (Sect. 3.2) que cette tentative répondait à un questionnement traditionnel concernant les problèmes diophantiens, savoir s'ils ont ou non une progressivité avérée, et, si oui, laquelle.

Je me propose ici de revenir sur cette tentative d'interprétation, pour en dégager les motifs et en critiquer rétrospectivement la méthode. Le but de cette retractatio est à la fois de mettre en valeur les différents sens dans lequel on peut selon moi qualifier certaines parties du texte de Diophante de séries de problèmes, et d'en tirer des réflexions plus générales sur l'intérêt qu'il y a à regarder cette démarche comme une forme de modélisation des textes pour l'étude des séries de problèmes du même genre, au sens où ce terme est entendu dans les travaux de W. McCarty $\left(2005\right.$, ch. 1) ${ }^{3}$. Plus précisément,

\footnotetext{
${ }^{2}$ Ma traduction s'inspire de celle de Ver Eecke (Diophante d'Alexandrie, 1926, p. 1), mais j'ai d'une part adopté une tournure assez fidèle à l'ordre des termes en grec, qui met fortement en évidence l'expression « l'invention des

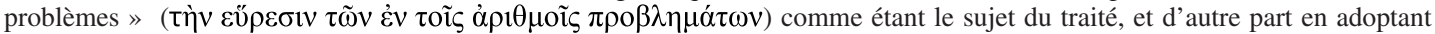
les conclusions de Christianidis (2007, pp. 4-5) ou encore de A. Allard (1980, p. 423) qui interprète à raison l'expression comme relative aux solutions des problèmes, non aux problèmes eux-mêmes.

3 Je dois la connaissance de ce travail et de son importance théorique à l'excellent séminaire sur l'édition numérique savante d'Aurélien Berra. Voir http://philologia.hypotheses.org/597 (consulté le $1^{\text {er }}$ Nov. 2015).
} 
nous voudrions ici montrer que l'établissement même de ce cadre analytique, visant à mettre en valeur la sérialité des problèmes diophantiens, représente une exploration méthodique du texte, reposant sur des hypothèses fondamentales, et sujette à révision dans la mesure où son approfondissement est aussi une façon d'explorer ses limites. C'est l'ensemble de ce processus heuristique qui permet de parler de «modélisation». Dans ce volume, cette lecture nous semble constituer un objet intéressant de réflexion méthodologique dans la mesure où il rejoint manifestement les préoccupations d'autres contributeurs au projet. Plusieurs d'entre eux sont en effet conduits eux aussi à interpréter la sérialité des textes ou des corpus qui les concernent à l'aide d'un choix de schématisation répondant à des hypothèses, et sujette à révision(s).

Pour aborder cette discussion, je commencerai pour cela par une brève présentation du texte et par quelques raisons qui invitent à en faire une «lecture rhétorique » (Sect. 2) : ce premier exposé me permettra d'expliquer deux sens dans lesquels on peut parler au sujet des problèmes diophantiens de « séries », l'un de ces sens étant explicité par Diophante lui-même dans la préface, le second provenant de l'historiographie (Sect. 3). Dans la quatrième partie je résumerai les principaux traits de l'interprétation de 2012, ce qui me permettra de souligner un troisième sens de la sérialité, qu'on pourrait qualifier de «sérialité reconstituée », au sens qu'elle dépend des choix méthodologiques initiaux. J'y évoquerai également les principales insuffisances de cette première interprétation et j'évoquerai les pistes de recherches sur le texte qui sont aujourd'hui les miennes, suite aux discussions au sein du groupe «séries de problèmes » (Sect. 4.4). Ce cheminement critique nous permettra de récapituler, en conclusion, les raisons qui permettent de qualifier ce travail d'interprétation de modélisation (Sect. 5).

\section{Le texte des Arithmétiques de Diophante et la structure des problèmes diophantiens}

\subsection{La sérialité déclarée des problèmes diophantiens}

Le texte ancien dont nous discutons ici différentes interprétations est un des principaux éléments du corpus mathématique en grec ancien, par son ampleur comme par le niveau très élevé de son contenu. L'auteur n'est connu que de nom, aucune indication crédible sur son identité, son niveau social et son environnement immédiat ne nous est parvenue ${ }^{4}$. On sait simplement qu'il a vécu antérieurement au $4^{\text {ème }}$ siècle EC, puisque Théon d'Alexandrie le cite et connaît ses travaux (Christianidis \& Skoura, 2013). De même, nous n'avons aucune idée réelle de la tradition textuelle qui conduit aux premiers témoins grecs connus dans le monde byzantin ou à la première traduction arabe d'une partie du texte. La préface du texte, dont l'authenticité n'est généralement pas mise en doute, précise que l'ouvrage initial était composé de treize livres de problèmes de nature arithmétique et qu'ils sont arrangés selon un ordre progressif au moins pour certains d'entre eux (Tableau 2) ${ }^{5}$.

\footnotetext{
${ }^{4}$ Il y a beaucoup de spéculations traditionnelles plus ou moins hasardeuses sur l'identité au moins culturelle de Diophante (Schappacher, 2005, pp. 5-7) ; des tentatives les plus sérieuses pour le situer précisément existent et remontent à P. Tannery, elles visent à le lier à deux personnages connus par ailleurs, Anatolius et son maître Dyonisius, mais ces identifications ne résistent pas vraiment à la critique (Diophante d'Alexandrie et Acerbi, 2011, p. 8 ; Knorr, 1993, p. 184).

${ }^{5}$ La traduction de ce passage est difficile, principalement en raison du sujet indéfini de la longue et complexe deuxième phrase.

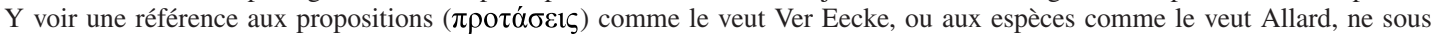
semble pas convaincant. Je suis ici l'interprétation adoptée pour notre traduction anglaise dans (Bernard \& Christianidis, 2012, p. 30), qui en fait une forme développée et «pluralisée » de la «matière » (Ű $\lambda \eta$ ) contenue ou « impliquée » dans les espèces.
} 
Tableau 2. Les dernières lignes de la préface des Arithmétiques (14.25-16.7).

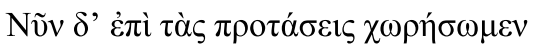

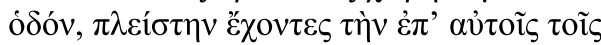

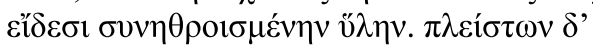

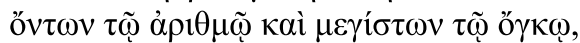

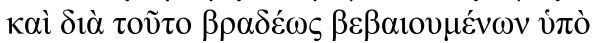

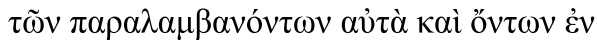

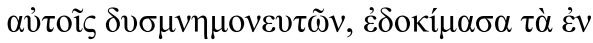

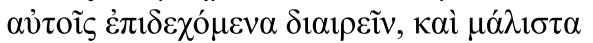

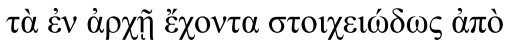

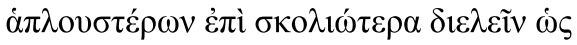

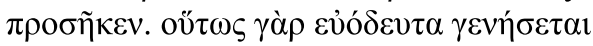

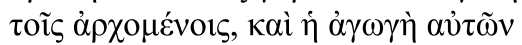

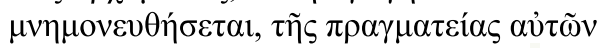

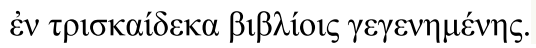

Dorénavant nous allons cheminer sur la voie des propositions, ayant réuni sur les espèces mêmes, une matière abondante. Comme ces choses sont très nombreuses et de grande ampleur et que, de ce fait, elles sont lentement maitrisées par ceux qui les abordent; et qu'il y a en outre en elles des choses difficiles à mémoriser, j'ai essayé de diviser ce qui, en elle, était susceptible de l'être, surtout pour séparer celles qui viennent au début, à la manière d'éléments, des plus tortueuses - comme il se doit. De la sorte, elles deviendront plus accessibles aux commençants, et leur méthode sera mémorisée. Quant au traitement complet, il comprend treize livres.

Ce second extrait donne d'une part une idée du style très elliptique par lequel Diophante donne en général, dans sa préface, des indications non seulement sur la méthode à suivre pour la résolution des problèmes du traité, mais encore sur l'arrangement de ces derniers. Plus précisément, il indique que le « contenu » de ces problèmes, qui est désigné de manière énigmatique dans tout ce passage et dont il est dit qu'il est lentement maîtrisé par les débutants, a été divisé et organisé, pour autant qu'il était possible de le faire, en allant des choses les plus simples vers les plus complexes, ou plus exactement les plus «tordues » $(\sigma \kappa o \lambda l \omega ́ \tau \varepsilon \rho \alpha)^{6}$. Quelle que soit l'interprétation qu'on peut faire de cet arrangement, question sur laquelle nous reviendrons plus loin, on peut parler ici d'une sérialité de premier degré, attribuable à Diophante lui-même, et qu'on pourrait qualifier de déclarée : le contenu des problèmes, ou du moins des plus simples d'entre eux, a fait l'objet d'un arrangement intentionnel.

\subsection{Les différentes parties de la préface du traité}

Parmi les treize livres explicitement annoncés par Diophante, six nous sont connus par la tradition directe, c'est-à-dire par une tradition manuscrite dont le plus ancien témoin remonte au 11ème siècle byzantin (Diophante d'Alexandrie et Acerbi, 2011, pp. 113-134; Perez Martin, 2006). Quatre autres livres, dont le contenu ne se confond pas, sauf pour quelques problèmes isolés, avec celui des « livres grecs », nous sont connus par une traduction arabe dont la rédaction remonte au 9ème siècle ${ }^{7}$ (Diophante d'Alexandrie, Qustā ibn Lūqā, \& Rāšid, 1984 ; Sesiano \& Diophante d'Alexandrie, 1982). Il manque donc trois livres à cet ensemble, non retrouvés jusqu'ici, pour reconstituer le tout. Sans m'arrêter ici aux nombreuses questions que posent cette double tradition, je me contenterai de quelques rappels généraux sur le contenu des premiers livres grecs. Ces derniers sont selon toute probabilité les trois premiers des treize livres originaux, et cette indication nous suffit ici car l'interprétation discutée ci-dessous ne concerne délibérément que ceux-là.

\footnotetext{
6 Je dois cette suggestion à Aurélien Berra, que sa fréquentation des problèmes plaisant de l'anthologie palatine ou des Deipnosophistes, a rendu sensible aux jeux d'esprits contenus dans certaines séquences d'énigmes.

${ }^{7}$ Le premier manuscrit connu est plus tardif, fin du 12è siècle.
} 
Tout d'abord, le texte commence par une longue préface à la fois originale et sophistiquée-au regard des autres préfaces des traités mathématiques classiques ${ }^{8}$. On peut subdiviser globalement le contenu de cette préface de la manière suivante :

(a) Un court préliminaire de quelques lignes, cité plus haut, définit la visée du traité dans des termes qui rappellent beaucoup ceux de la culture lettrée ancienne, fondée sur la rhétorique. En particulier, comme on l'a vu, Diophante précise qu'il entend répondre à l'ambition qu'il prête à son dédicataire, un certain Dionysios, de développer et d'apprendre une certaine capacité à inventer des solutions à de tels problèmes.

(b) Suit un court exposé sur la nature des nombres en général, qui se conclut par quelques constats fondamentaux sur la manière dont les problèmes arithmétiques sont «tressés » (plekesthai), c'està-dire constitués à partir de grandes catégories (comme les notions de nombres côté, carré, cube..) et d'opérations ou de rapports arithmétiques.

(c) Après une transition mettant en évidence que Diophante entend montrer une voie par laquelle le « tressage » de ces problèmes est « dénoué », c'est-à-dire par laquelle on peut résoudre les problèmes en question ${ }^{9}$, Diophante introduit un long exposé technique sur des nombres artificiels qui sont analogues, quant à leur fonction dans une solution, aux entités algébriques qui nous sont familières (l'inconnue et ses puissances). Ces nombres sont pourvus d'un mode de dénomination qui leur est propre, comme par exemple le terme arithmos, au sens d'un nombre inconnu dont on sert pour les solutions ${ }^{10}$, ou dunamis qui désigne son carré - du moins dans les termes qui nous sont aujourd' hui familiers ${ }^{11}$. Outre une nouvelle typologie de ces nombres artificiels et de leurs inverses, les opérations qu'on peut effectuer sur eux et sur leurs agrégats, sont longuement explicitées. C'est encore dans cette partie que le terme « d'espèce » (eidos) est introduit pour désigner ces types de nombres ou leurs agrégats.

(d) Un exposé complémentaire explique les opérations à appliquer spécifiquement aux équations qu'on est susceptible d'obtenir, au cours d'une solution, à partir des calculs dont Diophante a donné précédemment le principe. Cet exposé précise aussi les types d'équations qu'on peut principalement obtenir, à savoir les équations du type « une espèce égale une espèce », comme par exemple « deux dunameis, égales à 3 nombres $»^{12}$.

(e) Enfin la préface se conclut, comme on l'a vu (Sect. 2.1 et Tableau 2), par une conclusion plutôt énigmatique dont les termes et le niveau rappellent fortement la partie introductive (a) : elle explique en termes très généraux, et visiblement à titre de transition vers les problèmes eux-mêmes, comment ces derniers ont été intentionnellement arrangés dans un ordre progressif, destiné à faciliter l'apprentissage et la mémorisation, et mentionne l'existence de treize livres de problèmes.

\footnotetext{
8 Pour une vue d'ensemble, voir l'étude de (Vitrac, 2008) notamment, pour ce qui concerne Diophante, la conclusion (2008, p. 543-550) : la préface de l'ouvrage de Diophante y est rattachée à un ensemble d'ouvrages qui trahissent un souci pédagogique, tourné vers le lectorat des traités, bien plus marqué qu'à l'époque hellénistique.

${ }^{9}$ Il y a donc un jeu de mot explicite et très intéressant sur le couple plekein / luein, tresser / dénouer, le second terme pouvait aussi s'entendre naturellement comme renvoyant à la "solution" des problèmes. Sur ce couple plekesthai / luein, voir (Christianidis, 2007, Sect. 3.1).

${ }^{10}$ Dans nos traductions, nous traduisons ici par nombre, en italiques, de façon à garder la proximité avec le terme courant « arithmos », renvoyant à tout nombre quel qu'il soit, tout en signifiant la différence de nature par les italiques. Le nombre est l'analogue de notre inconnue « $\mathrm{x}$ » dans l'algèbre scolaire moderne.

${ }^{11} \mathrm{La}$ dunamis est donc l'analogue $\mathrm{x}^{2}$ de notre carré de l'inconnue x. Pour la liste complète de ces termes, voir Arithm. 4.12-6.21.

12 On verra ci-dessous, sur un exemple concret, à quoi renvoie cette partie de la solution (Sect. 2.3).
} 


\subsection{La structure et le schématisme des problèmes et de leurs solutions}

Poursuivons ces rappels généraux en évoquant la structure des problèmes que l'on trouve effectivement dans les livres grecs qui nous été conservés, notamment les cinq premiers, puisqu'ils suivent à l'évidence un schéma uniforme dont les étapes sont à peu près toujours respectées, moyennant les effets d'ellipse et les cas particuliers ${ }^{13}$. Pour simplifier l'exposé de ce schéma comprenant l'énoncé et la solution, il est plus simple de le décrire dans un premier temps à travers un exemple de problème à la fois banal et paradigmatique, que tel le cinquième du premier livre (Tableau 3) :

Tableau 3. Le schéma paradigmatique d'un problème diophantien (problème I.5).

\begin{tabular}{|c|c|}
\hline $\begin{array}{l}\text { Partager un nombre proposé en deux nombres de sorte que des } \\
\text { fractions données, mais différentes, de chacun des parties < du } \\
\text { nombre partagé }>\text {, une fois additionnées, fassent un nombre donné. }\end{array}$ & (1) Enoncé général \\
\hline $\begin{array}{l}\text { Le nombre donné doit toutefois être donné de telle façon, qu'ils soit } \\
\text { dans un endroit compris entre les deux nombres qu'on obtient en } \\
\text { prenant les fractions données, mais différentes, du nombre proposé au } \\
\text { début. }\end{array}$ & Diorisme \\
\hline $\begin{array}{l}\text { Qu'il soit donc prescrit de partager le }<\text { nombre }>100 \text { en deux } \\
\text { nombres, de sorte que le tiers du premier nombre }<\text { obtenu par le } \\
\text { partage }>\text { et le cinquième du second, additionnés ensemble, fassent } 30 \\
\text { unités. }\end{array}$ & $\begin{array}{l}\text { (2) Enoncé } \\
\text { «instancié »: les } \\
\text { nombres donnés sont } \\
\text { spécifiés. }\end{array}$ \\
\hline $\begin{array}{l}\text { Je pose le cinquième du second nombre, } 1 \text { nombre. Lui-même, par } \\
\text { conséquent, sera } 5 \text { nombres. Le tiers du premier sera donc } 30 \text { unités } \\
\text { en défaut d'un nombre; lui-même sera } 90 \text { unités en défaut de trois } \\
\text { nombres. Je veux, enfin, que les deux ensembles fassent } 100 \text { unités. } \\
\text { Mais les deux ensembles font } 2 \text { nombres et } 90 \text { unités. Ces dernières } \\
<\text { sont }>\text { égales à } 100 \text { unités. }\end{array}$ & $\begin{array}{l}\text { (3) } 1^{\text {ère }} \text { partie de la } \\
\text { solution, jusqu'à } \\
\text { l'équation }\end{array}$ \\
\hline $\begin{array}{l}\text { Et les semblables des semblables. Les restes sont donc10 unités, } \\
\text { égales à } 2 \text { nombres. Lenombre donc sera } 5 \text { unités. }\end{array}$ & $\begin{array}{l}\text { (4) } 2^{\text {ème }} \text { partie : de } \\
\text { l'équation à la valeur } \\
\text { du nombre. }\end{array}$ \\
\hline $\begin{array}{l}\text { Vers les valeurs. J'ai posé pour le cinquième du second, } 1 \text { nombre: } \\
\text { ce sera } 5 \text { unités, et lui-même sera donc } 25 \text { unités ; pour le tiers du } \\
\text { premier, } 30 \text { unités en défaut d'un nombre, ce sera } 25 \text { unités, } \\
\text { et lui-même sera } 75 \text { unités. }\end{array}$ & $\begin{array}{l}\text { (5) Calcul des valeurs } \\
\text { des nombres cherchés. }\end{array}$ \\
\hline $\begin{array}{l}\text { Et on a bien finalement que le tiers du premier avec le cinquième du } \\
\text { second }<\text { font }>30 \text { unités. }\end{array}$ & (6) Vérification, preuve \\
\hline
\end{tabular}

Ce schéma de la solution diophantienne typique, ce terme «typique » renvoyant à la fois à son caractère répétitif et à la présence concomitante de marqueurs textuels identifiables (soulignés dans le Tableau 3), peut donc se résumer en trois grandes étapes :

\footnotetext{
13 Ce schéma rappelle celui des propositions démonstratives classiques et de leurs parties, telles que Proclus de Lycie les identifie dans son commentaire au premier livre des Eléments d'Euclide. Pour une comparaison critique entre le schéma démonstratif classique, et la structure des solutions diophantiennes, voir la discussion détaillée proposée par F. Acerbi dans (Diophante d'Alexandrie et Acerbi, 2011, pp. 57-113) : si elle montre qu'un certain de degré de rapprochement est possible, il ne rend pas entièrement compte des particularités du style diophantien.
} 
- Une étape d'énonciation qui comprend les deux énoncés sous une forme générale (1) doublé souvent (mais pas toujours) d'une forme « instanciée » (2), ainsi que d'un diorisme ${ }^{14}$ en certains cas.

- Une étape de solution (3-4) qui se subdivise elle-même assez nettement en deux parties, dont la séparation est marquée par l'obtention d'une équation. Elle se conclut par l'obtention de la valeur du nombre, c'est-à-dire du nombre inconnu introduit pour les besoins de la solution.

- Une étape conclusive, par laquelle les valeurs des nombres cherchés sont déterminées à partir de la valeur trouvée de l'inconnue (5), puis une «preuve », c'est-à-dire une éventuelle vérification de la validité de ces résultats est faite, ou à tout le moins évoquée (6).

Toutes les étapes de ce schéma ne sont pas toujours présentes, ou pas au même degré. La seconde étape n'est pas toujours nécessaire car il n'y a pas toujours, dans l'énoncé général, de nombres donnés dont il faudrait spécifier la valeur. De même le diorisme n'apparaît-il que dans certains cas en nombre plutôt limité. La quatrième étape, de résolution de l'équation obtenue, est toujours nécessaire mais le texte grec en fait le plus souvent l'ellipse partielle (comme c'est le cas dans ce problème I.5) ou quasi-totale, ne conservant qu'une allusion à l'équation obtenue, puis la valeur du nombre inconnu sans donner aucun détail des calculs intermédiaires, qui sont effectivement faciles à reconstituer dans la plupart des cas ${ }^{15}$.

En outre, il faut encore noter que les problèmes en tant qu'unités textuelles, c'est-à-dire en tant qu'ensembles que la tradition manuscrite distingue par des espaces visibles, et que la version éditée distingue en outre par une numérotation, ne suivent pas tous un schéma aussi simple. S'ils débutent bien tous par un énoncé introduit par un verbe à l'infinitif, la solution peut avoir un degré d'élaboration qui conduit à énoncer des « sous-problèmes », notamment dans le cas où une première tentative de solution ne débouche pas sur les nombres cherchés mais sur une sorte d'impasse qui conduit à énoncer un nouveau problème auxiliaire (Bernard \& Christianidis, 2012, Sect. 3.3.5). Par contre le schéma exposé ci-dessus, même pour ces problèmes complexes décomposables en «sous-problèmes », garde tout son intérêt, puisqu'il permet d'analyser chacun de ces éventuels sous-problèmes de la même façon ${ }^{16}$.

\section{Le problème traditionnel posé par la progressivité des Arithmétiques}

\subsection{Des possibilités limitées d'interprétation comparative}

Ces rappels succincts et forcément schématiques suffiront cependant à expliquer les principales questions que soulève l'interprétation du contenu du traité en termes à la fois d'histoire culturelle et de cohérence entre ce qu'explique la préface, et ce que montrent les problèmes. Le premier problème est que nous n'avons que très peu d'éléments de comparaison peu ou prou contemporains de l'auteur ${ }^{17}$, qui puissent rendre compte de l'originalité de son contenu, notamment au niveau de la série de nombres « artificiels» introduits par Diophante pour les besoins de la solution

\footnotetext{
14 Un diorisme est une phrase précisant les conditions de possibilités du problème posé, c'est-à-dire les valeurs possibles pour les nombres donnés de l'énoncé, afin que le problème ait des solutions.

15 En l'occurrence dans notre exemple, 2 nombres et 90 unités égalent 100 unités, c'est l'équation obtenue. En enlevant « les semblables des semblables ", selon l'expression euclidienne consacrée, on obtient, en ôtant 90 unités de part et d'autre, 2 nombres égaux à 10 unités : c'est l'une des opérations sur les équations obtenues auxquelles la préface fait allusion. On obtient bien une équations du type «une espèce (des nombres) égale à une espèce (des unités) ». En divisant à nouveau par deux de part et d'autre, on obtient une équation qui permet de spécifier la valeur du nombre (son « hypostasis ») : 5 unités. Pour la traduction de hypostasis par « valeur», voir (Christianidis, 2015).

${ }^{16}$ C'est pourquoi le nombre des problèmes qui suivent le schéma ci-dessous, est légèrement supérieur aux nombres des problèmes traditionnellement reconnus sous ce terme, c'est-à-dire comme des unités textuelles. Voir le conspectus en annexe de (Bernard \& Christianidis, 2012, pp. 64-67) pour la correspondance détaillée entre les deux.

17 Dont la date, comme on l'a vu, n'est de toutes les façons pas connue.
} 
et dont il ne revendique manifestement pas la paternité ${ }^{18}$. La principale difficulté, de ce point de vue, est due au fait que nous n'avons pas, dans l'Antiquité, l'équivalent de ce dont disposent les collègues médiévistes tel Stéphane Lamassé : à savoir un large corpus de traités arithmétiques « au complet» dont on pourrait alors comparer la structure à celle du traité diophantien. A défaut, nous n'avons que les quelques épigrammes compilés dans l'Anthologie Palatine ou le témoignage de problèmes généralement isolés qu'on retrouve dans les papyri (Baillet, 1892 ; Fowler, 1999, pp. 234-238; 271-276) : cela n'autorise donc pas une comparaison à l'échelle de traités entiers, ou alors sur des problèmes de type très différents de ce qu'on trouve chez Diophante. Une comparaison plus prometteuse peut être faite au niveau linguistique et stylistique, en prenant comme point de comparaison le corpus des problèmes métrologiques comme ceux qu'on trouve dans les Métriques de Héron ou dans le corpus pseudo-héronien selon la voie suivie par B. Vitrac et F. Acerbi ${ }^{19}$. Si le thème mathématique de ces collections problèmes n'est pas le même que chez Diophante, les codes stylistiques employés présentent d'intéressantes parentés, nous y reviendrons en conclusion.

\title{
3.2 En termes de critique interne, le problème traditionnel posé par la sérialité déclarée
}

Concernant plus spécifiquement l'interprétation de la sérialité des problèmes de l'ouvrage diophantien, on s'est bien sûr interrogé de longue date, en écho à la revendication explicite de Diophante quant à l'arrangement intentionnel de ses problèmes (Tableau 2 supra), sur ce qui faisait dans le détail la progressivité et l'ordonnancement de la «matière » de ces problèmes. C'est ce qu'exprimait un jugement célèbre et désormais traditionnel de l'historien Hankel sur cette question :

\begin{abstract}
Il y a encore plus de diversité dans les solutions que dans les problèmes [de Diophante], et nous sommes complètement incapables de donner une vue d'ensemble raisonnablement exhaustive des différentes tournures de sa façon de procéder. De méthodes plus généralement compréhensives on ne trouve pas trace chez notre auteur : chaque question requiert une méthode spéciale, qui le plus souvent ne servira même pas pour les problèmes les plus apparentés. Il est ainsi difficile à un érudit moderne, même après qu'il a étudié 100 solutions diophantiennes, de résoudre le 101ème problème. (...) de cette façon, le lecteur court lui aussi avec une inquiétude intérieure de problème et en problème, comme dans un jeu d'énigmes, sans pouvoir jouir d'aucun. Diophante éblouit, plus qu'il ne satisfait l'esprit ${ }^{20}$.
\end{abstract}

On touche à un second sens de la sérialité diophantienne, directement issue de l'historiographie, et qui prend ici -tout jugement négatif mis à part- la forme d'une question : s'il y a bien une progressivité annoncée, où la trouve-t-on effectivement ? Comment l'induire de la lecture des problèmes et de leurs solutions, de manière pour ainsi dire «immanente » à la collection des problèmes, notamment les premiers puisque ce sont eux que Diophante dit avoir arrangés ?

Or cette question traditionnelle semble d'autant plus intéressante et pertinente, que cette revendication de progressivité est vraisemblablement liée à l'explicitation de la visée globale du traité, posée comme on l'a vu en termes de familiarisation progressive avec une méthode d'invention. Cette visée rappelle nettement les principes de la culture et de l'éducation lettrée antique, dont les idéaux de

\footnotetext{
18 La formule qu'il emploie (Arithm. 4.12) évoque plutôt un héritage tiré d'une littérature déjà connue de ses contemporains. Comme le rappelle Fabio Acerbi (Diophante d'Alexandrie, 2011, p. 10) ces dénominations se retrouvent dans les Métriques et dans la lettre de Psellus discutée par Tannery, quoiqu'avec un sens différent de celui que leur donne Diophante (Christianidis et Skoura 2013, n.40). En effet ce qu'on ne trouve pas ailleurs est la « mise en système » de ces dénominations dans une «théorie arithmétique », c'est-à-dire un système de nombres artificiels pourvus de règles de calculs.

${ }^{19}$ Pour ce type de littérature voir, dans ce volume, la contribution de B. Vitrac. Pour la méthode d'analyse linguistique proposée par les auteurs, voir par exemple (F. Acerbi, 2012 ; Héron d'Alexandrie, 2014, pp. 411-427).

${ }^{20}$ H. Hankel, traduit dans (Heath, 1910, pp. 54-55). Ma traduction.
} 
peut être tenu comme représentant une première interprétation du texte des trois premiers livres des Arithmétiques, en fonction de critères explicites qui permettent d'exhiber un ordre reconstitué, nous en présenterons ici plusieurs aspects utiles à notre discussion, à savoir le principe de cette interprétation, puis ses limites.

Le premier aspect important de ce travail était la nature du questionnement initial. Notre idée de départ était d'élargir la problématique de l'article de 2007 déjà mentionné, pour faire une hypothèse large sur la notion d'invention. Dans le contexte général où Diophante semble l'employer dans les premiers mots de la préface, il est en effet plausible qu'elle renvoie à une visée classique : celle de développer la capacité d'invention chez les auditeurs et apprentis en rhétorique, par un exercice répété et prolongé de « remâchage » sur un matériel pertinent. Dans cette interprétation il s'agirait donc d'une visée générale, un objet d'apprentissage et un «office » de l'apprenti-arithméticien, plus que d'un terme technique désignant une partie spécifique d'une solution diophantienne. En tout cas, cette interprétation posait une question non moins large sur le traité : en quoi la nature du projet cohérent qui semble soustendre les problèmes et leur progression revendiquée, répond-t-elle à un schéma d'organisation qu'on pourrait qualifier de rhétorique?

\subsection{Un rapide inventaire des traits « rhétoriques ॥ de la préface}

En fonction de ces prémisses, une première étape du travail a été de faire la liste des aspects du traité qui, explicitement ou implicitement, pouvaient se rapporter à la culture lettrée antique et à ses concepts fondamentaux, au-delà de la notion d'invention. Parmi ces grandes notions structurantes, on peut compter les suivantes :

(a) La notion d'imitation, au sens riche où l'entendait déjà Isocrate : comme un travail d'appropriation à partir d'un modèle. Dans la description que propose Diophante des rapports entre lui-même, comme maître, et son dédicataire impatient et désireux d'apprendre (supra, Tableau 1) - Dionysios, qui représente en général le lecteur - on reconnaît bien ce schéma fondamental de l'imitation. C'est possiblement ce modèle qui sous-tend également l'idée d'une voie, hodos, que Diophante dit montrer pour que le disciple et lecteur chemine en son long (Arithm. 4.10-11).

(b) La notion même de tâche, de problème (problêma) à laquelle il faut trouver une « solution », un dénouement qui n'épuise pourtant pas l'intérêt du problème. Il s'agit donc moins, visiblement, d'épuiser le sens d'une question en trouvant «les solutions », comme le voudrait un lecteur mathématicien moderne, que de montrer la façon de trouver des nombres qui satisfassent l'énoncé, à titre d'exercice de la faculté inventive. Ce sens est plus proche pour nous, d'une lecture théorique du contenu mathématiques des problèmes et de leurs solutions, que des usages pédagogiques toujours en vigueur, qui font du problème un terrain d'exercice. Ce sens est également proche du celui qu'Aristote donne à la notion dans ses Topiques, comme le rappelle B. Vitrac dans sa contribution au présent volume, pour opposer ce sens « aristotélico-diophantien » au sens des «problèmes » des Métriques, d'une nature essentiellement différente et pour lesquels Héron n'emploie d'ailleurs pas ce terme ${ }^{21}$.

(c) La notion de progressivité et de familiarisation graduelle, très nettement revendiquée par Diophante au début et à la fin de sa préface, et qui rappelle évidemment l'idée cardinale pour l'éducation lettrée antique, de « gymnastique de l'esprit » (gymnastics of the mind) pour reprendre l'expression suggestive de Rafaela Cribiore (Cribiore, 2001). L'invention se nourrit d'un « remâchage » des classiques $^{22}$ ainsi que de topiques guidant l'invention.

\footnotetext{
21 Voir dans ce volume l'article de B. Vitrac, partie II, « Le sens du terme problème ».

22 C'est cette référence à un «trésor » classique, qui fournit la matière primordiale de l'invention, qui différencie les démarches pédagogiques contemporaines des anciennes.
} 
(d) L'office de la mémoire, fait rarissime, est souligné par Diophante à la fin de sa préface : l'arrangement des problèmes est ainsi constitué, qu'il facilite la mémorisation (supra, Tableau 2). On retrouve une autre des fonctions cicéroniennes du rhéteur, déjà signalées dans l'Antiquité chez Aristote ou dans la Rhétorique à Alexandre, à côté de l'invention précisément.

Cette liste n'est pas exhaustive : elle donne simplement une idée d'un certain nombre de points de convergence possibles entre, d'une part, les concepts directeurs de l'éducation rhétorique antique, et d'autre part la structure du projet diophantien. Il faut noter d'emblée que tous ces rapprochements sont spéculatifs : nulle part, Diophante n'explicite totalement ce parallèle entre la culture lettrée et la structure de son traité. Il faut plutôt y voir les traces probables, ou encore les indices, d'une formation et d'une culture caractéristiques de l'élite lettrée dans l'Antiquité. A cette culture fortement imprégnée de rhétorique, la préface du traité fait donc des allusions marquées mais qui restent brèves. Quant au cœur du traité, c'est-à-dire dans les problèmes eux-mêmes, on trouve bien quelques commentaires sur la méthode, mais aucun qui puisse être relié de manière non ambiguë à la culture rhétorique. D'un autre côté, l'accumulation des indices relevés dans la préface invite à prendre au sérieux la tâche de reconnaître la mise en œuvre de ces principes dans la structuration et le style du traité lui-même.

\subsection{L'introduction d'un cadre d'analyse de la suite de problèmes, pour en reconstruire la sérialité possible}

C'est donc la notion déclarée de progressivité et d'arrangement des problèmes, qui nous invite à nous pencher de plus près sur le principe d'ordonnancement des problèmes diophantiens, tel qu'on peut le déceler à partir de l'organisation même du texte. C'est pour rendre compte de cette progressivité que J. Christianidis et moi-même avons entrepris d'élaborer un cadre analytique (Bernard \& Christianidis, 2012). Mon but ici n'est évidemment pas ici de revenir sur chaque détail de cette construction, mais d'en commenter quelques aspects essentiels qui permettent de comprendre à la fois la méthode, et d'en déceler les limites.

Examinons pour cela le type de tableau synthétique que nous avons systématiquement élaboré pour chacun des quelques 130 problèmes des trois premiers livres. Le schéma ci-dessous correspond au problème I.5 qui a été donné plus haut comme exemple (Tableau 5) :

Tableau 5. Schématisation du problème I.5 des Arithmétiques.

\begin{tabular}{|c|c|c|c|c|}
\hline \multicolumn{5}{|c|}{ ?X,Y: $100 \rightrightarrows X+Y\left(C_{1}\right), 1 / 3 X+1 / 5 Y \rightrightarrows 30\left(C_{2}\right)$} \\
\hline $\mathrm{P} \#$ & Positions & Method & Heuristic explanation & Remarks \\
\hline 1 & $1 / 5 \mathrm{Y}:=\mathrm{X}$ & simple 2 & & \\
\hline 2 & $\mathrm{Y}:=5 \mathrm{x}$ & Rw-d & & $\mathrm{P}_{1}$ \\
\hline 3 & $1 / 3 X:=30-x$ & Rw-d & & $\mathrm{P}_{1}, \mathrm{C}_{2}$ \\
\hline 4 & $X:=90-3 x$ & Rw-d & & $\mathrm{P}_{3}$ \\
\hline 5 & $X+Y:=2 x+90$ & Rw-d & loipon thelô $+\mathrm{C}_{1}$ & $\mathrm{P}_{1}, \mathrm{P}_{3}, \mathrm{C}_{1}$ \\
\hline & $\begin{array}{r}\text { Réd } \\
\text { Found } n \\
\text { Proof: ( }\end{array}$ & $\begin{array}{l}\text { on: } 2 \mathrm{x}+ \\
: 2 \mathrm{x}+90 \\
\mathrm{~s}: 25 \text { (fro } \\
75 \text { make }\end{array}$ & $\begin{array}{l}\mathrm{P}_{5} \text { and } \mathrm{C}_{1} \\
=10 \rightarrow \mathrm{x} \text { is } 5 \\
\text { et } 75\left(\mathrm{P}_{3} \text { and } \times 3\right) \\
-1 / 525 \text { make } 30\end{array}$ & \\
\hline
\end{tabular}

Pour l'essentiel, ce résumé schématise tout d'abord, à l'aide d'une traduction symbolique utilisant pour les nombres cherchés des lettres capitales, la procédure intrinsèque à l'un des deux énoncés, à savoir l'énoncé instancié (étape (2) de la traduction ci-dessus, supra Tableau 3). Le tableau quant à lui schématise la lère étape de la solution (étape (3) de la traduction), nous y revenons plus en détail ci-dessous. Les dernières lignes résument rapidement les étapes (4) à (6) : les opérations sur 
l'équation obtenue, les nombres effectivement trouvés et la vérification (la preuve). Le cœur de la schématisation porte donc en réalité sur la troisième étape, manifestement la plus importante dans la rédaction diophantienne ${ }^{23}$.

Le tableau résumant le cœur de la solution (étape 3), est quant à lui organisé autour d'un concept cardinal que nous avons retenu pour cette analyse, visible à l'entrée de chaque ligne du tableau et aux deux premières colonnes: il s'agit de celui de «position». Ce terme désigne la mise en correspondance d'un nombre auquel l'énoncé renvoie directement ou indirectement, et d'une expression formée des «nombres artificiels » introduits dans l'introduction (étape c) évoquée ci-dessus) ${ }^{24}$. Pour chacune de ces mises en correspondance est ensuite indiquée (troisième colonne) une « méthode d'invention », selon notre appellation, c'est-à-dire une manière typique d'obtenir de telles positions. "Typique » veut dire ici qu'elle est répétée, c'est-à-dire employée de manière récurrente au fil des problèmes ${ }^{25}$. Ici, par exemple, les positions 2 à 5 sont obtenues par une seule et même méthode labellisée « direct reworking », au sens que la nouvelle position est à chaque fois obtenue par des calculs simples, à partir des anciennes ${ }^{26}$. En général, le repérage et la classification de ces «types » de méthode forme le cœur de notre analyse. La colonne suivante « heuristic explanation » signale simplement la présence (ou non) d'explications explicites sur la méthode suivie, et la dernière indique soit les positions soit les conditions de l'énoncé prises comme référence pour l'établissement de la nouvelle position.

A partir de l'ensemble de ces tableaux, établis de manière systématique pour tous les problèmes identifiés dans les trois premiers livres, il nous était alors possible de constituer un tableau synoptique ${ }^{27}$, où les problèmes numérotés sont portés verticalement, et horizontalement le nombre de positions « utilisés » par chacun, ainsi surtout que la catégorisation de ces positions en fonction des « méthodes d'invention » employées ${ }^{28}$.

Le résultat intéressant est que, sur les 130 problèmes examinés sur l'ensemble des trois livres en question, il n'y a tout au plus qu'une dizaine de ces méthodes qui se trouvent employées et que nous avons toutes cherché à caractériser aussi précisément que possible. Si en outre on arrange ces dernières de gauche à droite, de la plus simple vers la plus compliquée, on voit assez nettement apparaitre une progressivité des problèmes dans les termes des méthodes utilisées. On peut alors interpréter cette progressivité en lien avec la sérialité « déclarée » dont il a été question plus haut (Sect. 2.1). En général il devient possible d'en tirer des conclusions positives quant à la possibilité même d'identifier une progressivité profonde des problèmes, puis de commencer à esquisser une interprétation de ce constat en termes rhétoriques ${ }^{29}$. Mais toute cette construction repose essentiellement sur une clé d'analyse qui reste à la fois implicite et discutable, à savoir cette notion de «position » comme unité d'analyse. Il s'agit donc d'une sérialité reconstituée.

\footnotetext{
23 Pour les étapes (4) à (6) en effet, il est caractéristique de cette rédaction que le détail est le plus souvent évoqué de manière elliptique, comme si le lecteur pouvait suppléer lui-même le détail des calculs.

24 Pour la notion de «position» telle que nous la comprenions alors, voir notre étude de 2012, (Bernard \& Christianidis, 2012, Sect. 2.4.3)

25 Pour une caractérisation plus précise, op.cit. Section 2.6.3.

26 Pour une définition plus détaillée illustrée par des exemples, voir (Bernard \& Christianidis, 2012, Sect. 3.2.2).

27 (Bernard \& Christianidis, 2012, pp. 64-68). Nous avons parlé au sujet de ce tableau de « conspectus » des problèmes diophantiens, par allusion aux conspectus habituels généralement fondés sur les énoncés des problèmes et qui ne donnent pas à voir la progression profonde de leurs solutions.

${ }^{28}$ La forme tabulaire n'est donc pas ici l'élément essentiel, il s'agit simplement d'une commodité pour permettre une récapitulation rapide. D'autres moyens auraient pu être envisagés, comme un système d'annotation uniforme par exemple. Ce qui est important ici n'est pas la forme, mais les critères d'analyse, par exemple la subdivision en une série de «positions ».

${ }^{29}$ Pour le détail de cette esquisse voir (Bernard et Christianidis 2012, 63-64). Un article est en préparation pour des remarques plus développées.
} 


\subsection{Les limites de ce cadre d'analyse}

Ce caractère reconstitué nous conduit du même coup naturellement au troisième aspect qu'il faut souligner sur ce travail d'élaboration d'un cadre analytique, et qui concerne maintenant les limites que nous n'avons pas tardé à lui reconnaître. Une des limites évidentes concernait la possibilité même de reconnaître pour toutes les positions la «méthode» par laquelle elles étaient obtenues. Dans certains cas en effet, la forme du texte, sa tournure allusive ou elliptique, ou ses accidents, nous conduisait à reconnaître des ambiguïtés rendant le choix difficile. Le point important était néanmoins que ces cas « limites » étaient en nombre restreint et ne mettaient pas en cause la robustesse globale du cadre proposé (2012, Sect. 4.4). D'autres limites, plus difficiles à cerner mais plus essentielles car remettant possiblement en cause le cadre d'interprétation, nous sont bientôt apparues. J'en résume ici quelques unes en reprenant l'exemple du problème I.5 :

(a) Dans notre analyse le dédoublement de l'énoncé (étapes (1) et (2), général et instancié) si particulier à Diophante, est comme oublié et «écrasé » par une écriture unique qui retranscrit généralement le seul énoncé instancié ${ }^{30}$. Le passage entre deux types d'énoncé, est donc mis « hors champ » dans notre cadre d'interprétation.

(b) Corrélativement, il y a une difficulté sur la manière précise dont les termes, désignant les nombres qui font l'objet d'une «position» (ou mise en correspondance, voir ci-dessus), sont effectivement et significativement rappelés de l'énoncé à la solution (ou d'une partie d'une solution à l'autre). Les modalités de ce rappel sont en effet également masquées par l'emploi d'un symbolisme qui oblitère leur construction langagière. Or ce point est important, car si les «nombres artificiels » ne sont employés que pour le cœur de la solution (étape (3)), les nombres donnés ou inconnus auxquels fait référence l'énoncé, par contre, font nécessairement l'objet d'un rappel dont la structure mérite d'être analysée ${ }^{31}$.

(c) Le fait de privilégier la mise en correspondance d'un nombre signifiant avec une expression de la théorie arithmétique, tend à oblitérer le contenu et le rôle de ce qui nous avons appelé les « explications heuristiques» par lesquelles ces correspondances sont obtenues. En particulier, le rôle très important des opérations et calculs arithmétiques dont la prescription est portée par ces explications, ne fait pas l'objet d'une analyse précise qui rendrait justice de leur importance pour l'économie globale de la solution. En réalité, dans notre étude, cette analyse est incorporée dans la description détaillée de chaque méthode, en référence à quelques exemples paradigmatiques seulement.

Ces limites sont très importantes, car il ne s'agit plus de simples ambigüités limitant l'application uniforme du modèle d'interprétation à tous les cas possibles, mais de problèmes beaucoup plus fondamentaux. Le fait en effet que soient oblitérés des aspects essentiels des solutions diophantiennes et de leur ancrage dans l'énonciation même des problèmes, contraint à repréciser le questionnement et à faire évoluer la grille d'interprétation. C'est pourquoi il apparaît aujourd'hui nécessaire de développer une nouvelle approche qui pallie ces défauts. Ce n'est pas le lieu ici de décrire en détail cette dernière, dont je finalise maintenant l'étude dans le cadre d'une nouvelle recherche portant précisément sur la manière dont la rédaction diophantienne relie énoncés et solutions par un jeu de variation sur les

\footnotetext{
30 C'est ce même procédé qu'emploi Stévin dans la traduction qu'il propose des problèmes diophantiens, dans son Arithmétique de 1585 (voir, dans ce volume, la contribution de J.M. Coquard). Chez lui il y a un problème générique, le 81 ème «Etant proposé question qui se solve par Algèbre : La solver par Algèbre » qui ouvre à toutes sortes d'exemples « diophantiens » qui ne se fondent en fait que sur l'énoncé instancié.

31 C'est précisément cette question générale du « pont » entre énoncés et solutions, pour ce type de problèmes d'allure « algébrique », qui a fait l'objet d'une de nos journées d'étude. Argumentaire détaillé : http://problemata.hypotheses .org/201 (consulté 5.10.15).
} 
procédures prescriptives qui constituent l'énoncé (Bernard, à paraître). Pour l'heure et pour conclure cette critique rétrospective de notre analyse des (premiers) problèmes diophantiens, j'en propose d'y reconnaître une forme de modélisation.

\section{La révision du cadre analytique, comprise comme un processus de modélisation}

Willard McCarty, dans son importante synthèse Humanities Computing (2005), défendait l'importance de considérer l'usage de moyens numériques en sciences humaines en général, et plus spécialement pour l'analyse de corpus littéraires complexes comme celle qu'il avait entreprise pour les Métamorphoses d'Ovide, comme une démarche intellectuelle et « humaniste » à part entière, à situer au même niveau que n'importe quel autre acte d'interprétation (2005, p. 3-6). Cette revalorisation des gestes techniques caractéristiques de l'édition et de l'analyse savante des textes par des moyens numériques, notamment les techniques d'encodage, passe pour lui par une critique de l'idée d'une « mise à disposition » de textes sous une forme facilitant ou rendant possible les recherches ${ }^{32}$ en faveur d'une défense de la codification numérique comme une démarche apparentée à la notion scientifique contemporaine de modélisation $^{33}$. Ce glissement fondamental supposait la mise en évidence de questions de recherches cardinales, et la reconnaissance fondamentale que l'intérêt d'une modélisation résidait davantage dans les choix opérés à la fois en amont qu'en aval du travail informatique, et pour l'aval par l'identification des limites des modèles proposés, que dans la « réussite» d'une mise à disposition d'un texte enrichi. Parler de modélisation, pour décrire ce processus heuristique, revient donc à tenter de décrire ce qui nous fait passer d'un certain état des questions à un autre, et c'est ce qui paraît le point le plus important.

C'est en référence aux réflexions suggestives de McCarty que nous proposons ici de parler d'un processus de modélisation du texte, en références au travail heuristique opéré en amont à partir d'un choix de concepts fondamentaux d'analyse, aboutissant en aval à une réflexion sur les limites fondamentales induites par ces choix, et à la nécessité d'une nouvelle recherche. C'est pour cette même raison que nous avons mis en évidence les questions cardinales qui ont présidé à l'établissement du modèle, ces questions étant fondamentalement ancrées sur des problématiques d'histoire culturelle : il s'agit en effet de faire sens au sein même du texte, des brèves et énigmatiques allusions à la culture rhétorique ancienne, contenues dans la préface (supra, Sect. 4.2). Pour le nouveau modèle dont j'étudie maintenant les linéaments, la question change, même si l'horizon de questionnement reste le même : il s'agit désormais d'étudier les modalités du lien entre langage des énoncés et structure des solutions, à l'aide d'une grille d'interprétation qui donne une place centrale au caractère procédural des énoncés. Ces nouvelles questions sont naturellement nées d'une exploration des limites de la première interprétation, dont le mode de schématisation ne permet pas encore d'atteindre le langage même et le "phrasé » très particulier et structuré des problèmes diophantiens (Sect. 4.4).

On retrouve de cette manière, quoiqu'à partir d'un questionnement de départ très différent, certaines préoccupations énoncés en conclusion de la contribution de S. Lamassé dans ce volume. Mais parce que cette nouvelle visée est plus proche d'une étude du langage diophantien, puisqu'il s'agit d'étudier la manière dont Diophante joue sur des variations de «formules fondamentales », elle se rapproche également des études séminales de F. Acerbi et B. Vitrac sur les différents « codes stylistiques » qui structurent la langue mathématique grecque, notamment ce qu'ils appellent les codes « algorithmiques » et surtout, « procéduraux », très employés par Diophante (Héron d'Alexandrie, 2014, pp. 411-427).

\footnotetext{
32 C'est ce qu'il appelle -par dérision- le knowledge jukebox, le « juke-box à connaissances ».

33 Cette analogie est défendue et justifiée dans le premier chapitre de l'ouvrage en question, particulièrement aux pages 37-53.
} 
Les séries de problèmes, un genre au carrefour des cultures

\section{Références}

Acerbi, F. (2012). I codici stilistici della matematica greca: dimostrazioni, procedure, algoritmi. Quaderni Urbinati di Cultura Classica CI(2). 167-216.

Allard, A. (1980). Diophante d'Alexandrie. Les Arithmétiques. Histoire du texte grec, édition critique, traductions et scolies. Louvain.

Baillet, J. (1892). Le Papyrus mathématique d'Akhmîn. Paris, France : Leroux.

Bernard. (2010). L'arrière plan rhétorique de la théorie de l'activité mathématique chez Proclus. In A. Lernould \& B. Vitrac (Eds.), Études sur le commentaire de Proclus au premier livre des «Éléments» d'Euclide (pp. 67-85). Villeneuve d'Ascq, France : Presses universitaires du Septentrion.

Bernard, A. (forthcoming). Arithmetical paraphrase in Diophantus's Arithmetica.

Bernard, A. (2003). Sophistic aspects of Pappus' Collection. Archive for the History of Exact Sciences 57/2: 93-150.

Bernard, A., \& Christianidis, J. (2012). A new analytical framework for the understanding of Diophantus's Arithmetica I-III. Archive for History of Exact Sciences, 66(1), 1-69. http://doi.org/10.1007/s00407-011-0090-5

Christianidis, J. (2007). The way of Diophantus: Some clarifications on Diophantus' method of solution. Historia Mathematica, 34(3), 289-305. http://doi.org/10.1016/j.hm.2006.10.003

Christianidis, J. (2015). The Meaning of Hypostasis in Diophantus' Arithmetica. In T. Arabatzis, J. Renn, \& A. Simões (Eds.), Relocating the history of science: essays in honor of Kostas Gavroglu (pp. 315-327). Cham, Suisse : Springer.

Christianidis, J., \& Skoura, I. (2013). Solving problems by algebra in late antiquity: New evidence from an unpublished fragment of Theon's commentary on the Almagest. SCIAMVS, (14), 41-57.

Cribiore, R. (2001). Gymnastics of the mind: Greek education in Hellenistic and Roman Egypt. Princeton.

Diophante d'Alexandrie. (1926). Diophante d'Alexandrie : les six livres arithmetiques et le livre des nombres polygones; oeuvres traduites pour la premiere fois du grec en français. (P. P. Ver Eecke, Ed.). Bruges, Belgique : Desclée, De Brouwer.

Diophante d'Alexandrie et Acerbi. (2011). De polygonis numeris. (éd. scient. et trad. F. Acerbi). Pisa, Italie.

Diophante d'Alexandrie, Qusțā ibn Lūqā \& Rāšid, R. Ḥafn̄̄. (1984). Les arithmétiques. Paris, France : Les Belles Lettres.

Fowler, D. (1999). The mathematics of Plato's Academy: a new reconstruction. Oxford, Royaume-Uni de Grande-Bretagne et d'Irlande du Nord : Clarendon Press.

Heath, T. (1910). Diophantus of Alexandria: a study in the history of Greek algebra. New York, EtatsUnis d'Amérique : Dover publications, 1964.

Héron d'Alexandrie. (2014). Metrica / Héron d'Alexandrie; introduction, texte critique, traduction française et notes de commentaire par Fabio Acerbi et Bernard Vitrac. (F. Acerbi \& B. Vitrac, Eds.) (Vol. 4). Pisa : Fabrizio Serra.

Knorr, W. R. (1993). Arithmêtikê stoicheiôsis: On diophantus and hero of Alexandria. Historia Mathematica, 20, 180-192. http://doi.org/10.1006/hmat.1993.1015

McCarty, W. (2005). Humanities computing. Basingstoke [England]; New York: Palgrave Macmillan.

Perez Martin, I. (2006). Maxime Planude et le Diophantus Matritensis: Un paradigme de la récupération des textes anciens dans la «Renaissance Paleologue. » Byzantion: Revue Internationale Des Études Byzantines, 76, 433-462.

Schappacher, N. (2005). Diophantus of Alexandria: a Text and its History. Version en ligne http://www-irma.u-strasbg.fr/ schappa/NSch/Publications_files/1998cBis_ Dioph.pdf (consult. 6.10.15) 


\section{SHS Web of Conferences}

Sesiano, J., \& Diophante d'Alexandrie. (1982). Books IV to VII of Diophantus' Arithmetica in the Arabic translation attributed to Qusțā ibn Lūqā. New York: Springer.

Vitrac, B. (2008). Promenade dans les préfaces des textes mathématiques grecs anciens. In Liber Amicorum Jean Dhombres (pp. 518-556). Brepols. Version en ligne https://hal.archives -ouvertes.fr/hal-00175172/document (consult. 6.10.15) 